\title{
Area Deprivation and Respiratory Morbidities in Children with Bronchopulmonary Dysplasia
}

\author{
Emma Banwell ${ }^{1}$, Joseph Collaco ${ }^{2}$, Gabriela Oates $^{3}$, Jessica Rice ${ }^{1}$, Lucia Juarez ${ }^{4}$, lisa \\ young $^{1}$, and Sharon McGrath-Morrow ${ }^{1}$ \\ ${ }^{1}$ University of Pennsylvania \\ ${ }^{2}$ Johns Hopkins Medical Institutions \\ ${ }^{3}$ University of Alabama at Birmingham \\ ${ }^{4}$ The University of Alabama at Birmingham School of Medicine
}

February 25, 2022

\begin{abstract}
Introduction: Infants and children diagnosed with BPD have a higher likelihood of recurrent hospitalizations and asthmalike symptoms. Socio-environmental factors that influence frequency and severity of pulmonary symptoms in these children during the pre-school age are poorly under-stood. In this study, we used the Area Deprivation Index (ADI) to evaluate the relationship between the socio-environmental exposures in children with BPD and respiratory outcomes during the first few years of life. Methods: A registry of subjects recruited from outpatient BPD clinics at Johns Hopkins University ( $\mathrm{n}=909)$ and the Children's Hospital of Philadelphia $(n=125)$ between January 2008 and October 2021 was used. Subjects were separated into tertiles by ADI scores aggregated to ZIP codes. Care-giver questionnaires were used to assess the frequency of respiratory morbidities and acute care usage for respiratory symptoms. Results: The mean gestational age of subjects was $26.8 \pm 2.6$ weeks with a mean birthweight of $909 \pm 404$ grams. The highest tertile (most deprived) of ADI was significantly associated with emer-gency department visits (aOR 1.72; $\mathrm{p}=0.009$ ), hospital readmissions ( $\mathrm{aOR} 1.66 ; \mathrm{p}=0.030$ ), and activi-ty limitations (aOR 1.55; $\mathrm{p}=0.048$ ) compared to the lowest tertile. No association was seen with steroid, antibiotic or rescue medication use, trouble breathing, or nighttime symptoms. Conclusion: In this study, children with BPD who lived in neighborhoods of higher deprivation were more likely to be re-hospitalized and have ED visits for respiratory reasons. Identifying socio-environmental factors that contribute to adverse pulmonary outcomes in children with BPD may provide opportunities for earlier interventions to improve long-term pulmonary outcomes.
\end{abstract}

\section{INTRODUCTION}

Preterm birth accounts for approximately $10 \%$ of live births in the United States annually and is a leading cause of mortality in children less than five years of age worldwide. ${ }^{1}$ Among infants born preterm, the most common serious complication is bronchopulmonary dysplasia (BPD), with almost 50,000 infants estimated to be diagnosed in the US annually. ${ }^{2}$ Although most infants with BPD will wean from respiratory support during the first few years of life, ${ }^{3}$ up to $50 \%$ will be re-hospitalized during that same period for pulmonary exacerbations, with many going on to develop asthma and asthma-like symptoms. ${ }^{4}$ Respiratory symptoms and abnormalities on pulmonary function testing, including chronic obstructive pulmonary disease, have been shown to persist into adulthood. ${ }^{5}$

With increasing numbers of preterm infants surviving into childhood and adulthood, identifying modifiable socio-environmental factors that influence long-term health outcomes in the outpatient setting may help optimize long-term clinical outcomes. ${ }^{6}$ In particular, factors that increase respiratory inflammation (such as infections, tobacco exposure, poor air quality, etc.) have been associated with worse lung function trajectories 
in patients with BPD. ${ }^{7,8}$ Socioeconomic disparities may expose certain infants and children to more of these risk factors. While individual risk factors can be assessed in isolation, many risk factors are co-related, with a potentially cumulative effect. Composite indices of socioeconomic disadvantage may allow for assessment of the cumulative risk associated with exposure to multiple community-level factors.

Various country-specific indices been used in previous studies to evaluate relationships between socioeconomic status and pediatric health outcomes, ${ }^{9}$ with significant associations being reported in a number of studies spanning several countries. Increased neighborhood deprivation has been shown to increase a child's risk of fractures ${ }^{10,11}$ obesity, ${ }^{12}$ abusive head trauma, ${ }^{13}$ and infant mortality. ${ }^{14}$ It has also been associated with worse outcomes for children with appendicitis, ${ }^{15}$ asthma, ${ }^{16-18}$ cystic fibrosis,${ }^{19}$ diabetes,${ }^{20}$ acute lymphoblastic leukemia, ${ }^{21}$ and after liver and kidney transplantation. ${ }^{22,23}$ In the United States, the Area Deprivation Index (ADI) measures the socioeconomic context of census block groups, which correspond to neighborhoods. The index is composed of 17 factors, including income, education, housing, and employment. ${ }^{9}$

Although previous research in BPD has evaluated outcomes based on single risk factors (e.g., insurance status), ${ }^{24,25}$ a recent French study showed that patients with BPD living in neighborhoods with high socioeconomic disadvantage had a three-fold higher risk of respiratory hospitalization compared to those living in affluent neighborhoods. ${ }^{26}$ A similar study has not yet been conducted in the United States. We hypothesized that greater neighborhood deprivation as quantified by the ADI would be associated adverse respiratory outcomes in a U.S. regional registry of infants and young children with BPD.

\section{METHODS}

Study Population: This study was conducted using a retrospective review of a database of subjects recruited from outpatient BPD pulmonary clinics at Johns Hopkins University $(n=911)$ and the Children's Hospital of Philadelphia $(\mathrm{n}=125)$ between January 2008 and October 2021. Two subjects in the Johns Hopkins University registry were excluded as ADI scores were not available for their zip codes to yield a study population of 1034 subjects. Inclusion criteria included prematurity ( $<37$ weeks gestation) and a diagnosis of BPD by NHLBI guidelines. ${ }^{27}$ This study was approved by the Johns Hopkins University Institutional Review Board (Protocol \#: NA_00051884; all caregivers consented) and the Children's Hospital of Pediatrics Institutional Review Board (IRB\# 20-017614; determined to meet exemption criteria).

Demographic and clinical data: Clinical data were obtained through chart review. Race and ethnicity were self-reported. Birth weight percentiles were derived from national U.S. data. ${ }^{28}$ Home oxygen and ventilator use were defined as use at the time of initial NICU discharge. Pulmonary hypertension was defined by the presence of pulmonary hypertension on echocardiogram on or after 36 weeks corrected age. ${ }^{29}$ Acute care for respiratory issues (occurrence of ER visits, hospitalizations, need for inhaled corticosteroids, and antibiotic use) and chronic symptom outcomes (trouble breathing, need for rescue medications, shortness of breath, nighttime symptoms) were collected through questionnaires at outpatient visits between ages 0-3 years.

Area deprivation scores: The 2019 ADI, created by the University of Wisconsin, is a validated measure of the socioeconomic context for U.S. census block groups, proxies for neighborhoods. ${ }^{9}$ The index (national scale from 0-100, with higher scores indicating worse deprivation) is constructed from 17 variables in the American Community Survey 5-year estimates. ADI scores for the study population were calculated using residential 5-digit ZIP codes and employing the 9-digit ZIP code crosswalk built to correspond to Census block groups. Median ADI scores were computed from all ADI values for block groups within each 5-digit ZIP code, excluding post office boxes, businesses, or large footprint entities, as done previously. ${ }^{19}$

Statistical methods: Subjects were separated into tertiles by median ADI scores for a zip code (ADI > 51.5 $(\mathrm{n}=345)$, ADI 32.5-51 $(\mathrm{n}=347)$, ADI $=<32(\mathrm{n}=342)$. Demographics and baseline clinical data of the highest and lowest tertiles were compared using chi square tests and t-tests. Associations between ADI tertiles and clinical outcomes were assessed using logistic regression mixed models adjusted for age at outcome data collection and potential demographic/clinical confounders (subjects with missing confounding variables were excluded from regression models); models were nested by individual and center. Sensitivity analyses were 
conducted using mean ADI scores for a zip code. All statistical analyses were conducted using Stata 17 (StataCorp; College Station, TX). $P$ values $<0.05$ were considered statistically significant.

\section{RESULTS}

Study population: Demographic and clinical characteristics for the study population $(\mathrm{n}=1034)$ are described in Table 1 . The study population was $43.1 \%$ female, $62.8 \%$ self-identified as non-White, and $5.5 \%$ self-identified as Hispanic. Mean gestational age at birth was $26.8 \pm 2.6$ weeks, and mean birth weight was $909 \pm 403$ grams. More than half $(53.4 \%)$ of the subjects were classified as having severe BPD, $33.4 \%$ moderate $\mathrm{BPD}$, and $13.2 \%$ mild $\mathrm{BPD}$. ZIP code ADI scores ranged from 5.5 to 93 , with a mean of 42.4 . There were differences in race, public insurance, gestational age, and birth weight by ADI scores. Subjects in the third ADI tertile (high deprivation) were more likely to be non-White $(p<0.001)$ and have public insurance $(p<0.001)$ than subjects in the first ADI tertile (low deprivation). Subjects in the most deprived ADI tertile also had earlier gestational age $(p<0.001)$ and lower birth weight $(p=0.007)$ than counterparts in the least deprived ADI tertile, but did not have later discharge ages $(p=0.12)$. The difference in birth weight by area deprivation may be attributable to the difference in gestational age, as birth weight percentiles did not significantly differ between ADI tertiles $(p=0.49)$. No differences were seen between the first and third ADI tertiles in terms of sex, ethnicity, birth weight percentile, BPD severity, and clinical characteristics at the time of NICU discharge, including home oxygen use, tracheostomy, home ventilator use, inhaled corticosteroid use, pulmonary hypertension, gastrostomy tube placement, and Nissen fundoplication. In terms of center differences, subjects receiving care at Johns Hopkins University had a lower median ADI (41.5 \pm 18.6$)$ compared to subjects receiving care at Children's Hospital of Philadelphia $(48.5 \pm 24.2 ; p<0.001)$, but the distribution of individuals in tertiles by center was not different $(p=0.06)$.

Clinical Outcomes: Data were collected from a total of 1800 caregiver questionnaires that surveyed subjects' needs for acute care for respiratory issues and their chronic symptoms. Logistic regression mixed models were used to determine the odds ratio of adverse outcomes for the most deprived versus the least deprived ADI tertiles, adjusting for age at outcome data collection, race, insurance type, and gestational age (Table 2 ). Models were nested by center to account for center-level differences. Higher neighborhood deprivation was significantly associated with emergency department visits (aOR 1.72; $p=0.009$ ), hospital readmissions (aOR 1.66; $p=0.030$ ), and activity limitations (aOR 1.55; $p=0.048$ ). No association was seen with steroid, antibiotic or rescue medication use, trouble breathing, or nighttime symptoms.

Sensitivity Analyses: Sensitivity analyses were carried using ZIP code mean ADI rather than median ADI. Similar to before, subjects receiving care at Johns Hopkins University had a lower mean ADI (42.0 \pm 17.6$)$ compared to subjects receiving care at Children's Hospital of Philadelphia (48.2 $\pm 21.8 ; p<0.001)$, but the distribution of individuals in tertiles by center was not different $(p=0.11)$. Results were similar overall, except a difference in discharge age and no difference in activity limitations between the first and third ADI tertiles (Supplemental Tables 1 and 2, respectively).

\section{DISCUSSION}

Our study examined the relationship between area socioeconomic deprivation and respiratory outcomes for infants with BPD using registry data. We found that infants and young children with BPD who lived in disadvantaged areas were more likely to have adverse respiratory outcomes including emergency department visits, re-hospitalizations, and activity limitations after adjusting for gestational age, race, and insurance status. These findings are consistent with prior studies that have linked deprivation with worse outcomes for a number of pediatric diseases. ${ }^{10-23}$ Compared to traditional socio-economic measures of individual income or insurance status, a potential advantage of area-level indices such as the ADI is their ability to capture community-level effects. Additionally, area-level indices provide an assessment of the joint effect of multiple risk factors, and this composite measure may be less likely to be influenced by anomalies in a single variable. ${ }^{30}$ Our study capturing multiple community-level factors assessed with the ADI illustrates that early-life community exposures are associated with poorer respiratory outcomes in preterm infants/children with BPD, likely contributing to health disparities in the very young. 
A French study using a socioeconomic deprivation index linked to census data found that the respiratoryrelated re-hospitalization rate was almost 3-fold higher for infants living in deprived neighborhoods (adjusted incidence rate ratio: $2.79 ; p<0.01) .{ }^{26}$ We observed a similar effect in our population (hospital readmissions adjusted OR 1.66; $p=0.030$ ). Neighborhood deprivation has been associated with adverse outcomes in other pediatric respiratory diseases as well. A national registry study found a significant association between ADI and respiratory outcomes in pediatric cystic fibrosis, specifically finding that children residing in the most deprived tertile had $2.8 \%$ lower forced expiratory volumes, 1.2 times more IV treatment nights, and a $20 \%$ higher risk of having more than 2 pulmonary exacerbations when compared to counterparts in the least deprived tertile. ${ }^{19}$ Similarly, studies of pediatric asthma have demonstrated associations between neighborhood deprivation and increased emergency calls for asthma exacerbations, ${ }^{16}$ and hospital readmissions for asthma, although the latter association is modified by health insurance coverage. ${ }^{17,18}$ It should be noted that not all studies of pediatric asthma have identified associations between the ADI and adverse outcomes. ${ }^{31}$

The connection between area deprivation and poorer outcomes in BPD is likely multifactorial. Neighborhoods with higher ADI scores may increase a patient's exposure to environmental risk factors for poor air quality, such as traffic pollution and tobacco smoke. ${ }^{32,33}$ Lower household income has been associated with increased risk for respiratory morbidities in prior studies. ${ }^{24}$ Income may be a limiting factor preventing families from finding housing with quality air ventilation, the lack of which has been associated with respiratory disease. ${ }^{34}$ Additionally, poverty, particularly inside cities such as Baltimore and Philadelphia, may be associated with residential crowding, which increases a child's potential exposure to respiratory diseases. ${ }^{35,36}$ Neighborhood deprivation may also be linked to decreased access to healthcare, which could make early outpatient intervention more difficult, leading to higher chances of requiring emergency care or hospitalization. Further studies will be necessary to delineate the pathophysiology behind this relationship.

In terms of factors that may play a role prior to NICU discharge, we found an association between deprivation and gestational age, suggesting that living in an area with high deprivation may increase the risk of preterm birth, similar to previous studies. ${ }^{37,38}$ There may also be NICU-level factors as well; although subjects in the most deprived tertiles were born at earlier gestational ages and lower birth weights, the length of initial hospitalization did not differ between first and third tertiles, nor did the frequency of use of medical technologies. It is unclear whether the similar initial hospitalization length and use of medical technologies reflects a "level playing field" at NICU discharge for all tertiles or whether subjects in the most deprived tertile may have less support at discharge than they could require based on gestational age and birthweight.

Limitations: A potential disadvantage of area-level indices is that neighborhood averages may not reflect the socioeconomic situation of families that may be outliers in their area, therefore individual patient situations should always be considered in the clinical setting. Additionally, based on data availability, ADI scores were aggregated to ZIP codes, with inevitable loss of geographic resolution and precision of estimates, especially in areas where poor and affluent neighborhoods are in close proximity. ${ }^{39}$ Patients enrolled in this study were from Baltimore and Philadelphia metropolitan areas, and results may not be generalizable outside of these geographic regions, or to patients not residing in the catchment area of a metropolitan tertiary care center. Our study population also has more severe respiratory disease (53\% met definition for severe BPD) compared to the preterm population as a whole (16\% of NHLBI validation cohort met criteria for severe $\mathrm{BPD}),{ }^{40}$ which may limit generalizability.

Conclusions: We found that area-level socioeconomic deprivation is associated with several poor respiratory outcomes in a contemporary cohort of infants and young children with BPD. Thus, assessment of area deprivation using the ADI may be a useful screening tool in an outpatient setting to identify young children with BPD at increased risk for adverse outcomes or to identify areas that would benefit from targeted public health interventions to improve respiratory health.

\section{REFERENCES}

1. Chawanpaiboon S, Vogel JP, Moller AB, Lumbiganon P, Petzold M, Hogan D, Landoulsi S, Jampathong N, Kongwattanakul K, Laopaiboon M, Lewis C, Rattanakanokchai S, Teng DN, Thinkhamrop J, Watananirun 
K, Zhang J, Zhou W, Gulmezoglu AM. Global, regional, and national estimates of levels of preterm birth in 2014: a systematic review and modelling analysis. Lancet Glob Health 2019;7(1):e37-e46.

2. Collaco JM, McGrath-Morrow SA. Respiratory Phenotypes for Preterm Infants, Children, and Adults: Bronchopulmonary Dysplasia and More. Ann Am Thorac Soc 2018;15(5):530-538.

3. Yeh J, McGrath-Morrow SA, Collaco JM. Oxygen weaning after hospital discharge in children with bronchopulmonary dysplasia. Pediatric pulmonology 2016;51(11):1206-1211.

4. Cheong JLY, Doyle LW. An update on pulmonary and neurodevelopmental outcomes of bronchopulmonary dysplasia. Seminars in perinatology 2018;42(7):478-484.

5. Collaco JM, McGrath-Morrow SA. Bronchopulmonary dysplasia as a determinant of respiratory outcomes in adult life. Pediatric pulmonology 2021.

6. Islam JY, Keller RL, Aschner JL, Hartert TV, Moore PE. Understanding the Short- and Long-Term Respiratory Outcomes of Prematurity and Bronchopulmonary Dysplasia. American journal of respiratory and critical care medicine 2015;192(2):134-156.

7. McGrath-Morrow SA, Collaco JM. Bronchopulmonary dysplasia: what are its links to COPD? Ther Adv Respir Dis 2019;13:1753466619892492.

8. Collaco JM, Aoyama BC, Rice JL, McGrath-Morrow SA. Influences of environmental exposures on preterm lung disease. Expert Rev Respir Med 2021;15(10):1271-1279.

9. Kind AJH, Buckingham WR. Making Neighborhood-Disadvantage Metrics Accessible - The Neighborhood Atlas. The New England journal of medicine 2018;378(26):2456-2458.

10. Evans S, Okoroafor UC, Calfee RP. Is Social Deprivation Associated with PROMIS Outcomes After Upper Extremity Fractures in Children? Clin Orthop Relat Res 2021;479(4):826-834.

11. Harrison WD, Chan YC, James LA, Nayagam S. The association of socioeconomic deprivation and paediatric open tibia fractures. J Pediatr Orthop B 2021;30(5):423-430.

12. Rossen LM. Neighbourhood economic deprivation explains racial/ethnic disparities in overweight and obesity among children and adolescents in the U.S.A. J Epidemiol Community Health 2014;68(2):123-129.

13. Beaulieu E, Jiang A, Zheng A, Rajabali F, Pike I. Inequities in Pediatric Abusive Head Trauma According to Neighborhood Social and Material Deprivation: A Population-Level Study in British Columbia, Canada. Child Maltreat 2020;25(3):300-307.

14. Yun JW, Kim YJ, Son M. Regional Deprivation Index and Socioeconomic Inequalities Related to Infant Deaths in Korea. J Korean Med Sci 2016;31(4):568-578.

15. Bogle R, McIntyre R, Patel N, Bradnock TJ. Deprivation increases perforation risk in paediatric appendicitis. Archives of disease in childhood 2021;106(4):392-393.

16. Laurent O, Filleul L, Havard S, Deguen S, Declercq C, Bard D. Asthma attacks and deprivation: gradients in use of mobile emergency medical services. J Epidemiol Community Health 2008;62(11):1014-1016.

17. Liu SY, Pearlman DN. Hospital readmissions for childhood asthma: the role of individual and neighborhood factors. Public Health Rep 2009;124(1):65-78.

18. Nkoy FL, Stone BL, Knighton AJ, Fassl BA, Johnson JM, Maloney CG, Savitz LA. Neighborhood Deprivation and Childhood Asthma Outcomes, Accounting for Insurance Coverage. Hosp Pediatr 2018.

19. Oates G, Rutland S, Juarez L, Friedman A, Schechter MS. The association of area deprivation and state child health with respiratory outcomes of pediatric patients with cystic fibrosis in the United States. Pediatric pulmonology 2021;56(5):883-890. 
20. Auzanneau M, Lanzinger S, Bohn B, Kroschwald P, Kuhnle-Krahl U, Holterhus PM, Placzek K, Hamann J, Bachran R, Rosenbauer J, Maier W, Initiative DPV. Area Deprivation and Regional Disparities in Treatment and Outcome Quality of 29,284 Pediatric Patients With Type 1 Diabetes in Germany: A Cross-sectional Multicenter DPV Analysis. Diabetes Care 2018;41(12):2517-2525.

21. Schraw JM, Peckham-Gregory EC, Rabin KR, Scheurer ME, Lupo PJ, Oluyomi A. Area deprivation is associated with poorer overall survival in children with acute lymphoblastic leukemia. Pediatric blood \& cancer 2020;67(9):e28525.

22. Wadhwani SI, Bucuvalas JC, Brokamp C, Anand R, Gupta A, Taylor S, Shemesh E, Beck AF. Association Between Neighborhood-level Socioeconomic Deprivation and the Medication Level Variability Index for Children Following Liver Transplantation. Transplantation 2020;104(11):2346-2353.

23. Chatelet V, Bayat-Makoei S, Vigneau C, Launoy G, Lobbedez T. Renal transplantation outcome and social deprivation in the French healthcare system: a cohort study using the European Deprivation Index. Transpl Int 2018;31(10):1089-1098.

24. Collaco JM, Choi SJ, Riekert KA, Eakin MN, McGrath-Morrow SA, Okelo SO. Socio-economic factors and outcomes in chronic lung disease of prematurity. Pediatric pulmonology 2011;46(7):709-716.

25. Lagatta J, Murthy K, Zaniletti I, Bourque S, Engle W, Rose R, Ambalavanan N, Brousseau D. Home Oxygen Use and 1-Year Readmission among Infants Born Preterm with Bronchopulmonary Dysplasia Discharged from Children's Hospital Neonatal Intensive Care Units. The Journal of pediatrics 2020;220:40-48 e45.

26. Deschamps J, Boucekine M, Fayol L, Dubus JC, Nauleau S, Garcia P, Boubred F. Neighborhood Disadvantage and Early Respiratory Outcomes in Very Preterm Infants with Bronchopulmonary Dysplasia. The Journal of pediatrics 2021;237:177-182 e171.

27. Jobe AH, Bancalari E. Bronchopulmonary dysplasia. American journal of respiratory and critical care medicine 2001;163(7):1723-1729.

28. Oken E, Kleinman KP, Rich-Edwards J, Gillman MW. A nearly continuous measure of birth weight for gestational age using a United States national reference. BMC pediatrics 2003;3:6.

29. Abman SH, Hansmann G, Archer SL, Ivy DD, Adatia I, Chung WK, Hanna BD, Rosenzweig EB, Raj JU, Cornfield D, Stenmark KR, Steinhorn R, Thebaud B, Fineman JR, Kuehne T, Feinstein JA, Friedberg MK, Earing M, Barst RJ, Keller RL, Kinsella JP, Mullen M, Deterding R, Kulik T, Mallory G, Humpl T, Wessel DL, American Heart Association Council on Cardiopulmonary CCP, Resuscitation, Council on Clinical C, Council on Cardiovascular Disease in the Y, Council on Cardiovascular R, Intervention, Council on Cardiovascular S, Anesthesia, the American Thoracic S. Pediatric Pulmonary Hypertension: Guidelines From the American Heart Association and American Thoracic Society. Circulation 2015;132(21):2037-2099.

30. Messer LC, Laraia BA, Kaufman JS, Eyster J, Holzman C, Culhane J, Elo I, Burke JG, O'Campo P. The development of a standardized neighborhood deprivation index. J Urban Health 2006;83(6):1041-1062.

31. Molina AL, Molina Y, Walley SC, Wu CL, Zhu A, Oates GR. Residential instability, neighborhood deprivation, and pediatric asthma outcomes. Pediatric pulmonology 2020;55(6):1340-1348.

32. Collaco JM, Aherrera AD, Breysse PN, Winickoff JP, Klein JD, McGrath-Morrow SA. Hair nicotine levels in children with bronchopulmonary dysplasia. Pediatrics 2015;135(3):e678-686.

33. Collaco JM, Morrow M, Rice JL, McGrath-Morrow SA. Impact of road proximity on infants and children with bronchopulmonary dysplasia. Pediatric pulmonology 2020;55(2):369-375.

34. Wimalasena NN, Chang-Richards A, Wang KI, Dirks KN. Housing Risk Factors Associated with Respiratory Disease: A Systematic Review. International journal of environmental research and public health $2021 ; 18(6)$. 
35. Evans GW, Saegert, S. Residential crowding in the context of inner city poverty. In: Wapner S, Demick, J., Yamamoto, T., Minami, H., editor. Theoretical Perspectives in Environment-Behavior Research. Boston, MA: Springer; 2000.

36. Colosia AD, Masaquel A, Hall CB, Barrett AM, Mahadevia PJ, Yogev R. Residential crowding and severe respiratory syncytial virus disease among infants and young children: a systematic literature review. BMC infectious diseases 2012;12:95.

37. O'Campo P, Burke JG, Culhane J, Elo IT, Eyster J, Holzman C, Messer LC, Kaufman JS, Laraia BA. Neighborhood deprivation and preterm birth among non-Hispanic Black and White women in eight geographic areas in the United States. Am J Epidemiol 2008;167(2):155-163.

38. Ncube CN, Enquobahrie DA, Albert SM, Herrick AL, Burke JG. Association of neighborhood context with offspring risk of preterm birth and low birthweight: A systematic review and meta-analysis of populationbased studies. Soc Sci Med 2016;153:156-164.

39. Durfey SNM, Kind AJH, Gutman R, Monteiro K, Buckingham WR, DuGoff EH, Trivedi AN. Impact Of Risk Adjustment For Socioeconomic Status On Medicare Advantage Plan Quality Rankings. Health Aff (Millwood) 2018;37(7):1065-1072.

40. Ehrenkranz RA, Walsh MC, Vohr BR, Jobe AH, Wright LL, Fanaroff AA, Wrage LA, Poole K, National Institutes of Child H, Human Development Neonatal Research N. Validation of the National Institutes of Health consensus definition of bronchopulmonary dysplasia. Pediatrics 2005;116(6):1353-1360.

\section{Hosted file}

ADI project tables 2-15-21.docx available at https://authorea.com/users/462413/articles/ 557841-area-deprivation-and-respiratory-morbidities-in-children-with-bronchopulmonarydysplasia 